\title{
Ripretinib in treatment of repeatedly relapsing rectal gastrointestinal stromal tumor: a case report
}

\author{
Chao Wu, Jun Zhang, Xingye Wu \\ Department of Gastrointestinal Surgery, The First Affiliated Hospital, Chongqing Medical University, Chongqing, China \\ Correspondence to: Xingye Wu. Department of Gastrointestinal Surgery, The First Affiliated Hospital, Chongqing Medical University, 1 Yixueyuan \\ Road, Chongqing 400016, China. Email: wuxingye19830221@126.com.
}

\begin{abstract}
Gastrointestinal stromal tumor (GIST) is the most common type of gastrointestinal mesenchymal tumor. Fewer than $2 \%$ of patients with metastatic GIST treated with imatinib experience a pathologic complete response. Furthermore, response to imatinib and subsequent-line tyrosine kinase inhibitor is limited by most patients developing drug resistance; median time to progression is 2 years for imatinib, and about half a year for sunitinib and regorafenib. In recent years, ripretinib, a fourth-line medicine, is been of the most important advances in the treatment of GIST. The ripretinib in patients with advanced gastrointestinal stromal tumours (INVICTUS): a double-blind, randomised, placebo-controlled, phase 3 trial study showed that ripretinib can significantly reduce the risk of disease progression and mortality in patients with advanced GIST $\geq 4$ lines, and has obvious clinical activity for a variety of KIT/ platelet-derived growth factor receptor $\alpha(P D G F R \alpha)$ gene mutations. This paper presents a case of advanced rectal GIST treated with ripretinib in China. In the years preceding, the patient did not respond well to first-third line agent therapies. However, a partial remission (PR) of the tumor was found after 3-courses of ripretinib treatment. For GIST patients with drug-resistant mutations (both primary and secondary), treatment may be a more accurate and reasonable when mutation-inhibitor agents are prescribed at an early stage.
\end{abstract}

Keywords: Case report; gastrointestinal stromal tumor (GIST); ripretinib; resistant mutation

Submitted Mar 03, 2021. Accepted for publication Apr 17, 2021.

doi: 10.21037/apm-21-722

View this article at: http://dx.doi.org/10.21037/apm-21-722

\section{Introduction}

Gastrointestinal stromal tumor (GIST) is the most common type of gastrointestinal mesenchymal tumor. It is reported that the incidence rate of GIST is about $10-15$ per million people (1). Due to it being insensitive to chemotherapy, surgical resection is still the main treatment of GIST. Repeat resection of postoperative relapsing GIST is not commonly performed as the prognosis is poor. The median survival time is only $6-18$ months, and the 5 -year survival rate is less than $10 \%$. It has been found that about $85 \%$ of primary GIST patients are caused by mutations in the protooncogene KIT or platelet-derived growth factor receptor $\alpha$ (PDGFR $\alpha$ ) of receptor tyrosine kinases (TKs). Since then, GIST became a model for the development of molecular-targeted therapy, which led to dramatically improved median overall survival of advanced GIST. But, further progress is needed after third-line or for TKI resistant mutations. The current second-line and third-line drugs are not good enough in the treatment of mutations in the active ring region, and the objective response rate is very low in patients with failed imatinib treatment (2).

Ripretinib is a potent switch pocket control inhibitor of KIT and PDGFR kinases and has activity against a broad range of mutations. It has recently demonstrated antitumoral activity across phase I to phase III clinical trials. Therefore, ripretinib has emerged as a new standard of care for advanced, multi-resistant GIST patients. Based on this data, the Food and Drug Administration has granted in 2020 the approval of ripretinib for GIST 
patients after progression to imatinib, sunitinib and regorafenib, is been of the most important advances in the treatment of GIST (3).

In this case, we prescribed ripretinib for a repeatedly relapsing rectal GIST patient, with KIT 11 and 17 mutations, who had not responded well to first-third line agents therapy for more than 10 years. A partial remission (PR) was observed after a few months, in which the tumor size was significantly reduced. We present the following article in accordance with the CARE reporting checklist (available at http://dx.doi.org/10.21037/apm-21-722).

\section{Case presentation}

The patient was a male who, at 34-years-old, presented with a pelvic mass on physical examination in 2008. He had no underlying diseases, no specific family or personal history, and an Eastern Cooperative Oncology Group (ECOG) score of 0 .

After admission, magnetic resonance imaging (MRI) showed an abnormal signal $(3.8 \times 4.0 \times 4.2 \mathrm{~cm})$ on the left side of the rectum in the pelvic cavity. In May 2008, under general anesthesia, exploratory laparotomy and local resection of the pelvic mass were performed. According to the results of HE staining and immunohistochemistry, leiomyoma and neurofibroma were excluded. The mass was defined as a rectal GIST and classified as high risk of recurrence and metastasis. Postoperative adjuvant therapy with imatinib (400 $\mathrm{mg}$ daily) was initiated and discontinued after intermittent dosing in the second year after surgery.

In December 2014, MRI reexamination revealed an abnormal shadow $(1.3 \times 1.0 \mathrm{~cm})$ in the left levator ani. Combined with the medical history, the local recurrence of GIST was considered. The patient underwent pelvic tumor resection in July 2015 in another hospital and GIST recurrence was confirmed. Gene testing showed a C-kit $11+17$ mutation. The patient restarted imatinib (400 mg daily) after surgery.

An MRI reexamination in November 2016 showed multiple abnormal signals on the left side of the rectum and sigmoid colon; the largest was $4.5 \times 3.6 \mathrm{~cm}$. Considering the failure of first-line drug therapy and tumor progression, treatment with the second-line drug sunitinib $(37.5 \mathrm{mg}$ daily) was started in the same month.

Third-line treatment and onwards after relapse: computed tomography (CT) examination in February 2018 suggested tumor progression; thus, treatment with the third-line drug regorafenib $(120 \mathrm{mg}, 3$ weeks on and 1 week off) was initiated in March 2018. Local interventions, microwave ablation, and other local treatments were also performed in another hospital. Pelvic recurrent GIST resection was performed in another hospital in November 2018. Postoperatively, the patient initiated oral imatinib (400 mg daily) in January 2019. In June 2019, pelvic GIST recurrence was detected again and the patient continued to be treated with oral imatinib (400 $\mathrm{mg}$ daily). The tumor slowly progressed and the patient developed perineal pain.

In July 2020, the patient was admitted to our center. Their general condition was good, no abnormalities were detected in biochemical tests, and the ECOG score was 1. An MRI examination showed multiple masses and nodules on the left side of the rectum; the largest was $4 \times 2.6 \mathrm{~cm}$ (Figure 1). After a multidisciplinary discussion, the patient was assessed as in the late stage of GIST recurrence. Despite local treatment, including surgery and tyrosine kinase inhibitor (TKI) medication, the disease still progressed, and it was determined that subsequent treatment should be based on systemic treatment with TKI medication. Then, the patient was evaluated for enrolment in "A single-arm, open-label, multicenter phase II clinical study to evaluate the efficacy, safety, and pharmacokinetic profile of DCC-2618 in patients with advanced GISTs that had progressed on prior therapy." Treatment with DCC2618 (ripretinib; $150 \mathrm{mg}$ po daily) was initiated in August 2020. The patient's mass began to shrink gradually and, after 3 cycles of treatment, PR was achieved. At present, the patient is on the ninth course of treatment and is tolerating the drug well, without serious drug-related side effects. The last MRI examination (Figure 1), in February 2021, showed that the largest tumor was $2.8 \times 1.7 \mathrm{~cm}$, which was evaluated as a PR to treatment (the total treatment process is shown in Figure 2).

All procedures performed in this study were in accordance with the ethical standards of the institutional and national research committees and with the Declaration of Helsinki (as revised in 2013). Written informed consent was provided by the patient.

\section{Discussion}

This patient was a typical case of rectal GIST with a primary KIT 11 mutation, who underwent complete resection of the primary lesion and adjuvant imatinib therapy in 2008 . According to the risk assessment, this was a case of highrisk GIST and, as recommended by current guidelines, the 

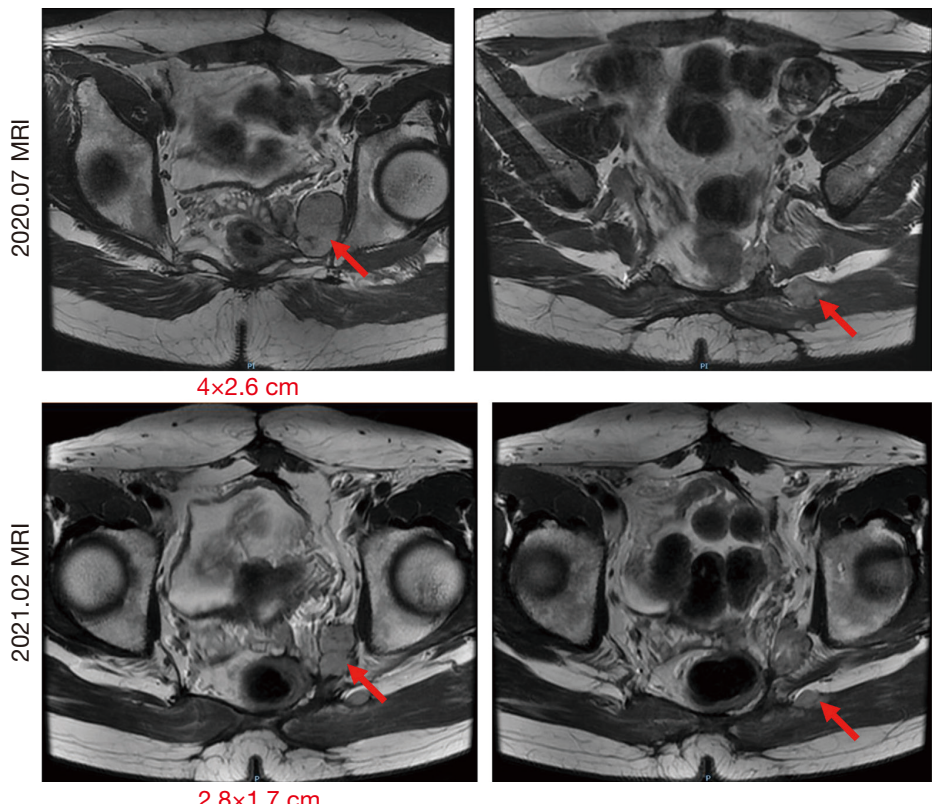

Figure 1 Assessing the tumor (red arrow) size by MRI before and after treatment of ripretinib. MRI, magnetic resonance imaging.

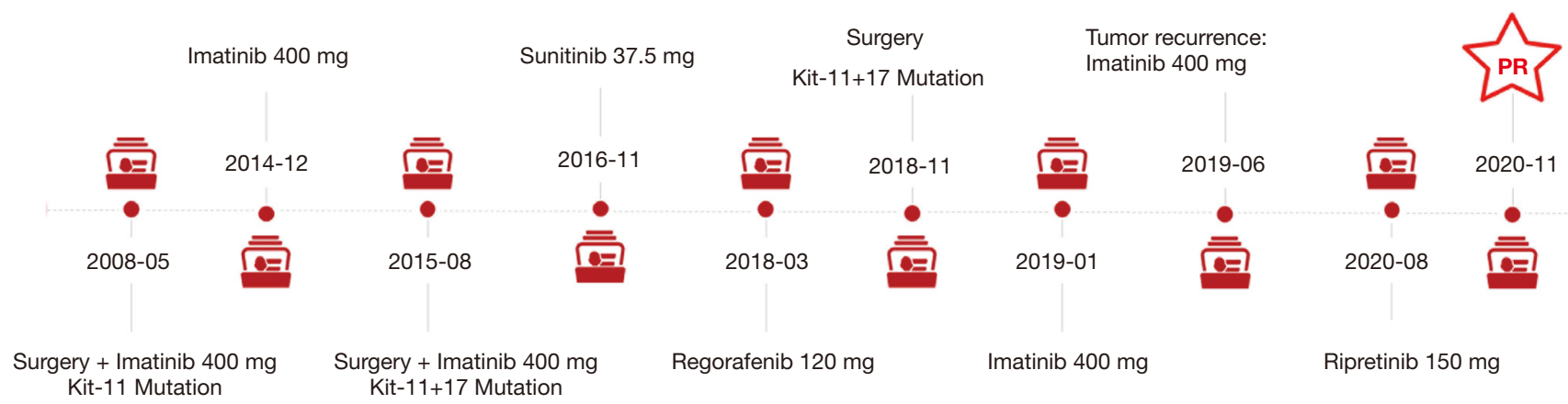

Figure 2 Treatment process of the patient.

duration of adjuvant imatinib therapy should have been at least 3 years. The patient developed local recurrence after 7 years, was treated again with surgery and imatinib, and the mutation type changed to primary KIT $11+$ secondary KIT 17. For patients with advanced GIST, the goal of metastasectomy is to remove disease before secondary resistance develops and to stop disease progression by eliminating resistant clones. The most retrospective studies (mostly single-institution) have demonstrated that cytoreductive surgery in patients with metastatic GIST is feasible, with greatest benefit in progression-free survival and overall survival observed in patients with responsive, stable, or limited disease progression on imatinib therapy.
But the benefit of metastasectomy for patients on sunitinib is difficult to assess (4).

The patient's disease relapsed and progressed again after 1 year of imatinib administration, and progressed again 1 year after switching to second-line sunitinib. The patient was then switched to third-line therapy combined with several rounds of local interventions, microwave ablation, and other local treatments, but the disease still progressed after 6 months. In retrospect, the course of progression might have been better if a drug more sensitive to secondary mutations had been chosen soon after the first relapse. After the failure of third-line therapy, there were no other more effective TKI drugs available at that time, and imatinib 
had to be administered again to slow the rate of disease progression; however, the tumor still grew slowly.

Thankfully, ripretinib, a new targeted therapeutic agent for GISTs, emerged in 2020. Ripretinib is a novel switch control inhibitor that has shown significant inhibitory effects on multiple mutation types of GIST (3). Data from the international multicenter phase III clinical study INVICTUS (5) showed that ripretinib has good clinical efficacy in patients with advanced GIST after $\geq 4$ lines of treatment, with an objective response rate of $11.8 \%$, median progression-free survival of 6.3 months, and median overall survival not reached. Based on the data from this study, ripretinib is now a standard fourth-line treatment for advanced GIST (6). This patient was already on fifth-line therapy when he received ripretinib. Additionally, the mass was relatively large and the patient was in poor physical condition. After the initiation of ripretinib, the patient's mass began to shrink gradually and, after 3 cycles of treatment, PR was achieved, which is a satisfactory response for a patient who has received multiple lines of therapy. Moreover, the safety and tolerability of ripretinib were relatively good (7), and the patient did not experience any serious adverse effects while taking the drug. The patient is currently in a good overall condition and continues to take ripretinib with the expectation of achieving longer-term benefits.

\section{Conclusions}

Ripretinib is a broad-spectrum TKI with good inhibitory effects on a wide variety of KIT/PDGFR $\alpha$ primary and secondary mutations. The international multicenter prospective clinical trial of ripretinib versus sunitinib in the second-line treatment of GIST has completed recruitment, and a second-line bridging study in China has been initiated. It is hoped that this study will address the treatment difficulties after first-line resistance and benefit patients in the future.

\section{Acknowledgments}

Funding: This work was supported by the Chongqing Science and Technology Committee (Grant No. cstc2015shm-szx120103), the Chongqing Science and Technology Bureau (Grant No. cstc2020jcyjmsxmX0360), Chongqing Health Committee (Grant No. 2020GDRC009), and High-level Medical Reserved Personnel Training Project of Chongqing.

\section{Footnote}

Reporting Checklist: The authors have completed the CARE reporting checklist. Available at http://dx.doi.org/10.21037/ apm-21-722

Conflicts of Interest: All authors have completed the ICMJE uniform disclosure form (available at http://dx.doi. org/10.21037/apm-21-722). The authors have no conflicts of interest to declare.

Ethical Statement: The authors are accountable for all aspects of the work in ensuring that questions related to the accuracy or integrity of any part of the work are appropriately investigated and resolved. All procedures performed in this study were in accordance with the ethical standards of the institutional and national research committees and with the Declaration of Helsinki (as revised in 2013). Written informed consent was provided by the patient.

Open Access Statement: This is an Open Access article distributed in accordance with the Creative Commons Attribution-NonCommercial-NoDerivs 4.0 International License (CC BY-NC-ND 4.0), which permits the noncommercial replication and distribution of the article with the strict proviso that no changes or edits are made and the original work is properly cited (including links to both the formal publication through the relevant DOI and the license). See: https://creativecommons.org/licenses/by-nc-nd/4.0/.

\section{References}

1. Blay JY, Kang YK, Nishida T, et al. Gastrointestinal stromal tumours. Nat Rev Dis Primers 2021;7:22.

2. Mohammadi M, Gelderblom H. Systemic therapy of advanced/metastatic gastrointestinal stromal tumors: an update on progress beyond imatinib, sunitinib, and regorafenib. Expert Opin Investig Drugs 2021;30:143-152.

3. Dhillon S. Ripretinib: First Approval. Drugs 2020;80:1133-8.

4. Fairweather M, Balachandran VP, Li GZ, et al. Cytoreductive Surgery for Metastatic Gastrointestinal Stromal Tumors Treated With Tyrosine Kinase Inhibitors: A 2-institutional Analysis. Ann Surg 2018;268:296-302.

5. Blay JY, Serrano C, Heinrich MC, et al. Ripretinib in patients with advanced gastrointestinal stromal tumours (INVICTUS): a double-blind, randomised, placebo- 
controlled, phase 3 trial. Lancet Oncol 2020;21:923-34.

6. Reiter A, George TI, Gotlib J. New developments in diagnosis, prognostication, and treatment of advanced systemic mastocytosis. Blood 2020;135:1365-76.

7. Florou V, Trent JC, Wilky BA. Precision medicine

Cite this article as: Wu C, Zhang J, Wu X. Ripretinib in treatment of repeatedly relapsing rectal gastrointestinal stromal tumor: a case report. Ann Palliat Med 2021;10(4):4994-4998. doi: 10.21037/apm-21-722 in gastrointestinal stromal tumors. Discov Med 2019;28:267-76.

(English Language Editor: J. Jones) 